\title{
Inverse Kinematics for a Point-Foot Quadruped Robot with Dynamic Redundancy Resolution
}

\author{
Alexander Shkolnik and Russ Tedrake \\ Computer Science and Artificial Intelligence Laboratory \\ Massachusetts Institute of Technology, Cambridge, MA 02139 \\ \{shkolnik, russt\}@mit.edu
}

\begin{abstract}
In this work we examine the control of center of mass and swing leg trajectories in LittleDog, a point-foot quadruped robot. It is not clear how to formulate a function to compute forward kinematics of the center of mass of the robot as a function of actuated joint angles because point-foot walkers have no direct actuation between the feet and the ground. Nevertheless, we show that a whole-body Jacobian exists and is well defined when at least three of the feet are on the ground. Also, the typical approach of work-space centering for redundancy resolution causes destabilizing motions when executing fast motions. An alternative redundancy resolution optimization is proposed which projects single-leg inverse kinematic solutions into the nullspace. This hybrid approach seems to minimize 1) unnecessary rotation of the body, 2) twisting of the stance legs, and 3) whole-body involvement in achieving a step leg trajectory. In simulation, this control allows the robot to perform significantly more dynamic behaviors while maintaining stability.
\end{abstract}

\section{INTRODUCTION}

The Jacobian is a powerful tool to linearize the inverse kinematics of a robot, and can be used to achieve velocity commands in the direction of the correct kinematic solution. In redundant systems, such as many quadrupeds and humanoid robots, the nullspace of the Jacobian spans the infinite solutions that define the gradient of the specified task. Redundancy resolution is often attempted by finding a solution that tends to bring the system to some desirable position, e.g. workspacecentering. In this work we examine the inverse kinematics redundancy resolution problem for LittleDog, a point-foot quadruped robot developed by BostonDynamics.

Typically, Jacobians are calculated by differentiating the forward kinematics of the system. However, for a point-foot walker, there is no actuation at the ankle, therefore no direct control over the foot angle with the ground. It is not clear how to specify the forward kinematics defining the center of body (COB) position as a function of actuated joint angles. The derivation for the whole-body Jacobian of the COB is therefore not trivial. One approach to control the body position is to treat each leg as a separate robotic arm, and move each leg in the opposite direction of the desired body motion. This single-leg inverse kinematic solution was found to perform reasonably well in simulation, but the approach suffers from a limited workspace as it does not take advantage of rotations of the body that could extend reach. In this work we show that the whole-body COB Jacobian does exist, and is well defined when three or four of the feet are on the ground. Whole-body Jacobians associated with the tasks of controlling 1) center of mass (COM) trajectory and 2) swing foot trajectory are derived based on the COB Jacobian. A moving robot by definition violates the assumptions of static stability. Using the usual method of work-space centering for redundancy resolution with a whole-body Jacobian seems to compound the problem, as it utilizes all limbs to execute any motion producing more joint movement than necessary. In simulation, this caused the robot to fall over fairly easily when executing fast movements. It turns out that projecting the single-leg inverse kinematic solution into the nullspace of the whole-body Jacobian produces a solution which improves the dynamic stability of the system. We refer to this approach as dynamic redundancy resolution. This hybrid approach seems to minimize 1) unnecessary rotation of the body, 2) twisting of the stance legs, and 3) whole-body involvement in achieving a step leg trajectory. This offers a significant performance improvement over either approach taken separately, as measured experimentally in simulation.

\section{BACKGROUND}

Achieving stable locomotion over irregular terrain has proven to be a challenging problem. The main difficulty comes from the fact that legged robots are inherently underactuated, as there is limited control of the body position and orientation. The DARPA Learning Locomotion project sponsors several development teams with the intent of developing a robust walking controller to enable a position controlled quadruped robot, LittleDog, to traverse very rough terrain by applying machine learning algorithms.

Point-foot walkers, such as LittleDog, have the advantages that it is easier to select placements for feet contacts on the terrain, and significantly simplifies simulation, as one does not have to worry about the surface of the foot. However, point-foot walkers exemplify the underactuation problem. In addition, LittleDog has many redundant degrees of freedom (DOF); each leg can act to push the body in different directions, so care must be taken to coordinate the actuators of all legs. Foot planning and maintaining stability margins are crucial for the success of a walking vehicle, but lower level inverse kinematics algorithms must do the necessary work of coordinating available actuators while also maximizing stability. Further, the lower level control can be useful for reducing the dimensionality of the system. LittleDog has 18 DOF, including six unactuated degrees specifying body 
position and orientation, a two DOF universal joint at each hip and a hinge joint at each knee. The sheer number of DOF in this system can be prohibitive for many learning tools. To achieve a crawl gait, one may attempt to control the robot whole-body center of mass and the position of a foot. This cuts the controlled degrees of freedom to six, or potentially even fewer ${ }^{1}$, and makes learning algorithms more plausible.

Walking and even standing-humanoid controllers typically take into account some metric of stability. The notion of true stability for locomotion is difficult to quantify precisely (see [1]), so heuristics are often utilized for this purpose. These often include: maximizing the static stability margin [2], maximizing the ZMP margin [3], Resolved Momentum Control (RMC) [4], and Zero Spin Center of Pressure (ZSCP) Control [5], etc (see [6] for review). Essentially all of these approaches try to control the center of mass of the entire robot, so we pay particular attention to this issue in this work. This is true even for ZMP, where a COM trajectory can be computed to implement a desired ZMP trajectory [7].

The RMC framework for humanoid robots attempts to control a humanoid robot's whole-body linear and angular momentum (or components of these), using a framework similar to the whole-body Jacobian, while constraining feet movement to specified trajectories [4]. Linear momentum divided by mass of the system translates to COM velocity, so the high level objective of RMC is similar to this work. However, RMC assumes actuated ankles, and is primarily concerned with how to utilize free DOFs of a humanoid, for example the arms and torso, to help keep the robot stable. The quadruped robot does not have such flexibility. Further, the work developed here allows for hierarchical control with priority given to the COM velocity rather than to the feet velocities, whereas RMC does not allow for this.

This paper is organized as follows: 1) Background 2) Derivations of the partial inverse kinematics control of the COB; 3) Derivations of the whole-body Jacobians associated with the tasks COM control and swing foot control; 4) Results are presented from running three resulting controllers in simulation, and limited results are presented from experiments on the actual robot.

\section{Single-Leg Inverse Kinematics Control}

A first approach to developing a walking controller for the position controlled walking robot might be to treat all of the legs separately, and control the center of body and the orientation of the body by moving the legs appropriately. When considering each leg separately, we utilize the relation:

$$
\mathbf{F} \mathbf{P}_{G}=\mathbf{X}_{B_{4}}+\mathbf{R}_{B} \cdot \mathbf{F} \mathbf{P}_{L}
$$

where $\mathbf{F P}_{G} \in \mathbb{R}^{3 \times 4}$ contains the feet positions in the global frame; $\mathbf{F P}_{L} \in \mathbb{R}^{3 \times 4}$ contains the feet positions in the robot

\footnotetext{
${ }^{1}$ Note, in this framework it is intuitive to further reduce dimensionality in higher level controllers, for example by constraining the height of the $\mathrm{COB}$ or COM. The swing leg may also be constrained to operate in a plane, reducing the system to only $4 \mathrm{DOF}$.
}

relative frame; $\mathbf{X}_{B} \in \mathbb{R}^{3}$ is the center of body position and $\mathbf{X}_{B_{4}} \in \mathbb{R}^{3 \times 4}$ is $\left[\mathbf{X}_{B} \mathbf{X}_{B} \mathbf{X}_{B} \mathbf{X}_{B}\right] ; \mathbf{R}_{B} \in \mathbb{R}^{3 \times 3}$ is the rotation matrix of the body. Differentiating and solving for the feet velocities in the relative frame we obtain:

$$
\dot{\mathbf{F P}} \dot{P}_{L}=\mathbf{R}_{B}^{T} \cdot\left(\dot{\mathbf{F P}} \dot{\mathbf{X}}_{G}-\dot{\mathbf{X}}_{B}-\dot{\mathbf{R}}_{B} \cdot \mathbf{F} \mathbf{P}_{L}\right)
$$

Note that $\mathbf{F} \mathbf{P}_{G}$ is assumed to be zero for stance feet, and is otherwise the velocity command of the swing foot. $\dot{\mathbf{X}}_{B}$ and $\dot{\mathbf{R}}_{B}$ are the commanded COB and orientation velocities.

For each leg, i, we may compute the single-leg Jacobians, $\mathbf{J}_{l e g_{i}} \in \mathbb{R}^{3 \times 3}$, of the foot position w.r.t. the robot frame. The joint velocities corresponding to each leg can then be computed:

$$
\dot{\mathbf{q}}_{l e g_{i}}=\mathbf{J}_{l e g_{i}}^{-1} \cdot \mathbf{F} \dot{P}_{L_{i}}
$$

The results is a control for COB, not COM. Further, it may be useful to allow the body rotation to be left unspecified. This increases the workspace of the system by allowing the robot to rotate the body to help reach places that would otherwise be kinematically infeasible. To deal with these issues, we utilize whole-body Resolved Motion Rate Control [8].

\section{Whole-Body JACOBIAN CONTROL}

In general, forward kinematics, transforming joint angles $\mathbf{q} \in \mathbb{R}^{n}$ into some task space $\mathbf{x} \in \mathbb{R}^{m}$, and the differentiation of this relation is given by:

$$
\begin{aligned}
\mathbf{x} & =f(\mathbf{q}) \\
\dot{\mathbf{x}} & =\mathbf{J}(\mathbf{q}) \cdot \dot{\mathbf{q}}
\end{aligned}
$$

where $J(q) \in \mathbb{R}^{m \times n}$ is the whole-body Jacobian associated with the task space of $\mathbf{x}$. The inverse kinematics with nullspace optimization for redundancy resolution can be solved as in [9]:

$$
\dot{\mathbf{q}}=\mathbf{J}^{+} \cdot \dot{\mathbf{x}}+\alpha\left(I-\mathbf{J}^{+} \cdot \mathbf{J}\right) \cdot \dot{\mathbf{q}}_{r e f}
$$

where $\mathbf{J}^{+}=\left(\mathbf{J}^{T} \mathbf{J}\right)^{-1} \cdot \mathbf{J}^{T}$ is the Moore-Penrose pseudoinverse of $\mathbf{J}, \alpha$ is a scalar weighting, and $\dot{\mathbf{q}}_{r e f} \in \mathbb{R}^{n}$ is a low priority command in joint space. For the redundant case where $m<n$, the solution, $\dot{\mathrm{q}}$ should achieve the commanded $\dot{\mathrm{x}}$ while also minimizing $\left\|\dot{\mathbf{q}}-\dot{\mathbf{q}}_{\text {ref }}\right\|$.

Note, we may have multiple tasks, and control them in a hierarchical manner [10], for example:

$$
\begin{aligned}
\dot{\mathbf{q}}_{1} & =\mathbf{J}_{\text {low }}^{+} \cdot \dot{\mathbf{x}}_{\text {low }}+\alpha_{1}\left(I-\mathbf{J}_{\text {low }}^{+} \cdot \mathbf{J}_{\text {low }}\right) \cdot \dot{\mathbf{q}}_{\text {ref }} \\
\dot{\mathbf{q}} & =\mathbf{J}_{\text {high }}^{+} \cdot \dot{\mathbf{x}}_{\text {high }}+\alpha_{2}\left(I-\mathbf{J}_{\text {high }}^{+} \cdot \mathbf{J}_{\text {high }}\right) \cdot \dot{\mathbf{q}}_{1}
\end{aligned}
$$

where $\mathbf{x}_{\text {high }}$ represents a "high priority task" with associated $\mathbf{J}_{\text {high }}$ Jacobian, and conversely $\mathbf{x}_{\text {low }}$ is a "lower priority task" with associated $\mathbf{J}_{\text {low }}$ Jacobian. $\dot{\mathbf{q}}_{1}$ is the joint level command that would be assigned by the low priority task, and is passed on as a "suggested" command to the high priority task.

Typically, the lowest priority task in joint space consists of specifying $\dot{\mathbf{q}}_{r e f}$ to move down a potential to bring the posture to some standard "favored" position, as in [11]. Note that it is possible to differentiate (5), which would allow for control at the acceleration or force level (for review see [12]). Because the LittleDog robot is position and velocity controlled, we will limit our discussion here to controllers that resolve kinematic redundancies at the velocity level. 


\section{A. Jacobian of Center of Body}

Here we attempt to compute the Jacobian, $\mathbf{J}_{X_{B}}(q)=\frac{\partial \mathbf{X}_{B}}{\partial \mathbf{q}_{a}}$, of the center of body, $\mathbf{X}_{B} \in \mathbb{R}^{3}$, where $\mathbf{q} \in \mathbb{R}^{18}$ describes all 18 degrees of freedom, and $\mathbf{q}_{a} \in \mathbb{R}^{12}$ is the actuated subset of q, not including the 6 unactuated degrees of freedom of the body position and orientation.

The typical approach for computing the Jacobian would be to define forward kinematics and then differentiate w.r.t. actuated joint angles. It is not obvious how to do this for the center of body of the robot, and some assumptions must be made. First, note that given $\mathbf{q}$, we may compute the position of all feet in both relative and absolute coordinate systems. Given absolute positions of three feet, and three leg lengths, we may compute the position of the center of body by performing trilateration, e.g. by finding the intersection of three spheres, centered at the feet positions with radii corresponding the distance between the foot and COB. However, this derivation is bulky and, is not well defined for cases with four feet.

In order to get around the problem of underactuation, we specify that the feet on the ground do not move. Consider what happens when changing the leg lengths; first note that the leg lengths, $L \in \mathbb{R}^{4}$, are simply the distance between the center of body and the feet position. This is defined in either the global coordinate frame, or the robot coordinate frame for each foot, i:

$$
\begin{aligned}
L_{i} & =\left\|\mathbf{F} \mathbf{P}_{L_{i}}\right\| \\
& =\left\|\mathbf{X}_{B}-\mathbf{F P}_{G_{i}}\right\|
\end{aligned}
$$

Let us assume that all feet are on the ground (otherwise use the subset of three feet which are on the ground), and the feet are not moving: $\frac{\partial \mathbf{F} \mathbf{P}_{G}}{\partial \mathbf{q}_{a}}=0$. Then we differentiate $\mathrm{L}$ w.r.t. $\mathbf{q}_{a}$ :

$$
\begin{aligned}
\frac{\partial L}{\partial \mathbf{q}_{a}} & =\frac{\partial L}{\partial \mathbf{F} \mathbf{P}_{L}} \frac{\partial \mathbf{F} \mathbf{P}_{L}}{\partial \mathbf{q}_{a}} \\
& =\frac{\partial L}{\partial \mathbf{X}_{B}} \frac{\partial \mathbf{X}_{B}}{\partial \mathbf{q}_{a}}
\end{aligned}
$$

Note that all of these derivatives are well defined, and have geometrically understandable meanings. For example, $\frac{\partial L}{\partial \mathbf{X}_{B}}$ is the change of leg lengths given a movement of the body (imagine a table with springs for legs; the springs change length in a well defined way if the table is moved around or rotated). We can now solve for $\frac{\partial \mathbf{X}_{B}}{\partial \mathbf{q}_{a}}$ using the Moore-Penrose pseudoinverse:

$$
\frac{\partial \mathbf{X}_{B}}{\partial \mathbf{q}_{a}}=\left[\frac{\partial L}{\partial \mathbf{X}_{B}}\right]^{+} \frac{\partial L}{\partial \mathbf{F} \mathbf{P}_{L}} \frac{\partial \mathbf{F} \mathbf{P}_{L}}{\partial \mathbf{q}_{a}}
$$

where

$$
\begin{aligned}
& \frac{\partial L}{\partial \mathbf{X}_{B}}=\frac{\partial\left\|\mathbf{F} \mathbf{P}_{G}-\mathbf{X}_{B}\right\|}{\partial \mathbf{X}_{B}} \\
& =\frac{\mathbf{X}_{B}-\mathbf{F P}_{G}}{\sqrt{\left\|\mathbf{X}_{B}-\mathbf{F P} \mathbf{P}_{G}\right\|}} \\
& \frac{\partial L}{\partial \mathbf{F} \mathbf{P}_{L}}=\frac{\partial\left\|\mathbf{F} \mathbf{P}_{L}\right\|}{\partial \mathbf{F} \mathbf{P}_{L}} \\
& =\frac{\mathbf{F P}_{L}}{\sqrt{\left\|\mathbf{F} \mathbf{P}_{L}\right\|}} \\
& \frac{\partial \mathbf{F P}_{L}}{\partial \mathbf{q}_{a}}=\left|\begin{array}{cccc}
\mathbf{J}_{l e g_{1}} & 0 & 0 & 0 \\
0 & \mathbf{J}_{l e g_{2}} & 0 & 0 \\
0 & 0 & \mathbf{J}_{l e g_{3}} & 0 \\
0 & 0 & 0 & \mathbf{J}_{l e g_{4}}
\end{array}\right|
\end{aligned}
$$

\section{B. Jacobian of Body Rotation}

By Differentiating (1) w.r.t. $\mathbf{q}_{a}$, for the stance feet, and assuming that these feet are not slipping so that $\frac{\partial \mathbf{F} \mathbf{P}_{G}}{\partial \mathbf{q}_{a}}=0$, we find that:

$$
\frac{\partial \mathbf{R}_{B}}{\partial \mathbf{q}_{a}} \cdot \mathbf{F} \mathbf{P}_{L}=-\frac{\partial \mathbf{X}_{B}}{\partial \mathbf{q}_{a}}-\mathbf{R}_{B} \cdot \frac{\partial \mathbf{F} \mathbf{P}_{L}}{\partial \mathbf{q}_{a}}
$$

The Moore-Penrose pseudoinverse is applied to solve for $\mathbf{J}_{R_{B}}=\frac{\partial \mathbf{R}_{B}}{\partial \mathbf{q}_{a}}:$

$$
\mathbf{J}_{R_{B}}=-\left(\frac{\partial \mathbf{X}_{B}}{\partial \mathbf{q}_{a}}+\mathbf{R}_{B} \cdot \frac{\partial \mathbf{F} \mathbf{P}_{L}}{\partial \mathbf{q}_{a}}\right) \cdot\left(\mathbf{F} \mathbf{P}_{L}\right)^{+}
$$

\section{Center of Mass Jacobian}

The conversion from local coordinates (L) to global coordinates $(\mathrm{G})$ for the center of mass, $\mathbf{X}_{M}$ is specified by:

$$
\mathbf{X}_{M_{G}}=\mathbf{X}_{B}+\mathbf{R}_{B} \cdot \mathbf{X}_{M_{L}}
$$

Then we can solve for $\mathbf{J}_{X_{M G}}=\frac{\partial \mathbf{X}_{M_{G}}}{\partial \mathbf{q}_{a}}$ :

$$
\mathbf{J}_{X_{M G}}=\frac{\partial \mathbf{X}_{B}}{\partial \mathbf{q}_{a}}+\mathbf{R}_{B} \cdot \frac{\partial \mathbf{X}_{M_{L}}}{\partial \mathbf{q}_{a}}+\frac{\partial \mathbf{R}_{B}}{\partial \mathbf{q}_{a}} \cdot \mathbf{X}_{M_{L}}
$$

\section{Swing Foot Jacobian}

The conversion from relative to global coordinates for the position of a specific foot, $\mathbf{X}_{S W}$, is specified by:

$$
\mathbf{X}_{S W_{G}}=\mathbf{X}_{B}+\mathbf{R}_{B} \cdot \mathbf{X}_{S W_{L}}
$$

Then we can solve for $\mathbf{J}_{X_{S W G}}=\frac{\partial \mathbf{X}_{S W_{G}}}{\partial \mathbf{q}_{a}}$ :

$$
\mathbf{J}_{X_{S W_{G}}}=\frac{\partial \mathbf{X}_{B}}{\partial \mathbf{q}_{a}}+\mathbf{R}_{B} \cdot \mathbf{J}_{\operatorname{leg}_{S W}}+\frac{\partial \mathbf{R}_{B}}{\partial \mathbf{q}_{a}} \cdot \mathbf{X}_{S W_{L}}
$$

where $\mathbf{J}_{\operatorname{leg}_{S W}}$ is the swing-foot Jacobian relative to the body. 


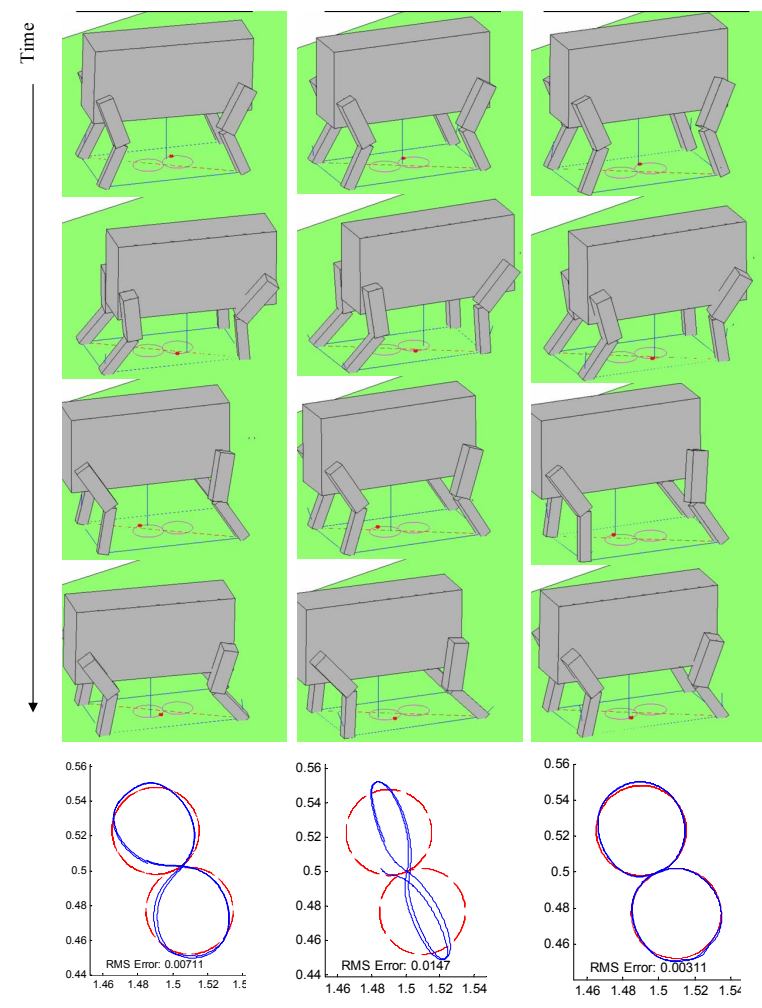

Fig. 1. Simulation Results: Case 1. COM figure-8 Trajectory with four legs. Left: Partial IK. Performance is reasonably good. Center: Whole-body Jacobian with Work centering. The centering appears to interfere with this task. Note, it is possible to reduce gains on the centering, but this results in worse performance in other tests. Right: Hybrid approach. The trajectory following appears very good, and results in the lowest RMS Error. Bottom: red line is the commanded trajectory ( 2 seconds), blue line is the actual trajectory.

\section{E. Hierarchical Controller}

As shown in (7), the Jacobians and associated nullspaces can be "stacked" in a hierarchical manner. Given the goal of enabling stable walking over rough terrain, it is logical to give the control of $\dot{\mathbf{X}}_{M_{G}}$ the highest priority. Of second priority, then, is $\dot{\mathbf{X}}_{S W_{G}}$. The final step in developing the full control law is choosing an appropriate $\dot{\mathbf{q}}_{r e f}$ to plug into (7). Workspace centering is established by choosing $\dot{\mathbf{q}}_{r e f}$ along the gradient of the potential: $\left\|\mathbf{q}_{a}-\mathbf{q}_{0}\right\|$ for some favored position, $\mathbf{q}_{0} \in \mathbb{R}^{12}$.

A proposed alternative to workspace centering is to compute the partial IK solution, as in section III:

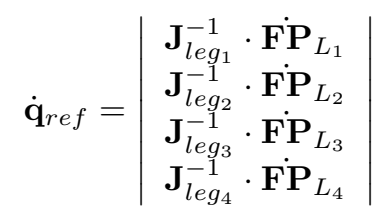

Note that using the single-leg inverse kinematics represents a dynamic redundancy resolution method, as opposed to the static method of workspace centering which always tries to move the robot back to the $\mathbf{q}_{0}$ position.
The final control is specified by:

$$
\begin{aligned}
\dot{\mathbf{q}}_{1} & =\mathbf{J}_{S W_{G}}^{+} \cdot \dot{\mathbf{x}}_{S W_{G}}+\left(I-\mathbf{J}_{S W_{G}}^{+} \cdot \mathbf{J}_{S W_{G}}\right) \cdot \dot{\mathbf{q}}_{r e f} \\
\dot{\mathbf{q}} & =\mathbf{J}_{M_{G}}^{+} \cdot \dot{\mathbf{x}}_{M_{G}}+\left(I-\mathbf{J}_{M_{G}}^{+} \cdot \mathbf{J}_{M_{G}}\right) \cdot \dot{\mathbf{q}}_{1}
\end{aligned}
$$

Note, kinematic joint limits can also be included as a task. This control is only activated when joints are close to their limits, and is thus not included here for clarity.

\section{RESUlTS}

\section{A. Simulation}

In this section we explore the performance of these three controllers by looking at a simulation of a quadruped robot with parameters similar to the LittleDog robot. The physics based dynamics simulation was constructed with SDFast, and the controller was implemented in Matlab. A springdamper ground model is utilized with point-feet contacts, with reasonable ground reaction forces. The simulation has 4 testcases to illustrate the performance in sample COM and swing foot trajectory tasks.

- CASE 1: Figure 8 trajectory for COM, with 4 feet on ground. In this case, a figure 8 desired trajectory is tracked with the whole robot center of mass. Four feet remain on the ground.

- CASE 2: Figure 8 trajectory for COM, with 3 feet on ground. In this case, a figure 8 desired trajectory is tracked with the whole robot center of mass. Three feet remain on the ground, and one foot is raised in the air.

Figure 3 shows the ZMP and center of mass, comparing the work-centering and hybrid approaches. The wholebody Jacobian with work-centering utilizes more drastic motions, with the involvement of all limbs, which results in the ZMP straying far from the COM projection. This undermines the static stability assumption that we made when selecting the figure $8 \mathrm{COM}$ trajectory to follow, and results in the robot eventually toppling over. On the other hand, the ZMP corresponds fairly well with the COM projection in the hybrid controller, as this controller minimizes drastic movements. Thus, even though the robot is moving fairly quickly (executing the figure 8 motion in 3 seconds), the static stability margin is reasonable to use with the controller using the dynamic redundancy resolution. This illustrates why the hybrid controller is more stable when executing fast movements.

- CASE 3: Small, fast, figure 8 trajectory for swing leg. In this case, a small figure 8 is to be tracked by the front right leg, while the COM is to remain over the centroid of the support polygon. In this test, the entire figure 8 trajectory is completed quickly (in $1 \mathrm{sec}$.).

- CASE 4: Very large, slower, figure 8 trajectory for swing leg. In this case, a very large figure 8 , requiring the robot to pitch its body up and down to complete it accurately, is to be tracked by the front right leg, while the center of mass is to remain over the centroid of the support polygon. In this test, the figure 8 trajectory is completed fairly slowly, over a period of 15 seconds. 


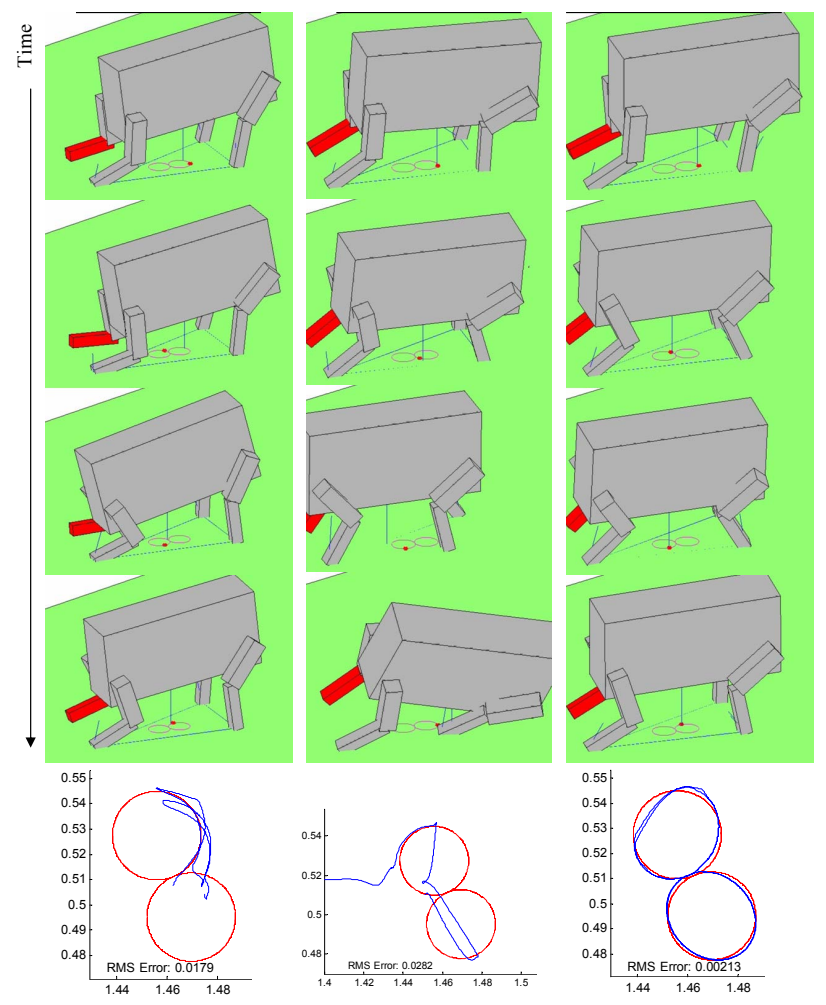

Fig. 2. Simulation Results: Case 2. The red leg designates the swing foot in the air. Left: Partial IK. The robot is unable to follow the specified trajectory at the speed given.Center: Whole-body Jacobian with Work centering. The robot can not finish the task as he rotates forward and eventually flips over. Right: Hybrid approach. This is the only approach that is able to follow the trajectory even somewhat closely. Bottom: red line is the commanded trajectory ( 3 seconds), blue line is the actual trajectory.

\section{B. Real Robot}

Joint trajectories were generated by using the simulator with the hybrid controller for the test cases presented above. These trajectories were recorded, and passed to the actual LittleDog robot as a feedforward command. The performance in cases 13 was quite good, and resembled the performance seen in the simulators. Case 4 , where joint-limits play a larger, role still requires some more work. When we tried to run the controller using feedback on the real dog, Case 1 (with all four feet on the ground) looked good. The other cases, which involve a foot in the air, did not perform well because stance feet were slipping, which violated our Jacobian assumptions that grounded feet were not moving. This was enhanced by oscillations due to feedback latencies. We are working on further developing the feedback controller; for the purpose of walking, we are also working on generating open-loop trajectories between each step, so that feedback is incorporated between steps, while each step is executed using the feedforward trajectory generated with the hybrid controller.

\section{CONCLUSIONS}

This work presents derivations for whole-body Jacobians for center of mass and swing foot trajectory control of a point-foot quadruped robot, despite the fact that the forward
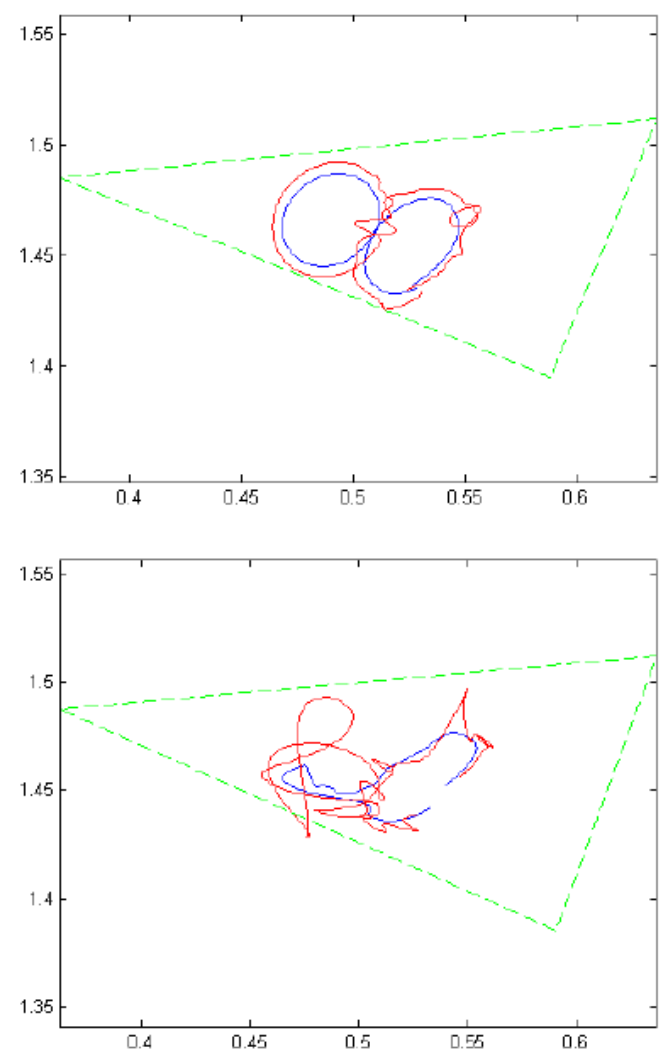

Fig. 3. ZMP (red) vs COM (blue) for Case 2 with work-centering (bottom) and hybrid control (top). The triangle depicts the support polygon. This figure illustrates that ZMP more closely follows the COM trajectory in the hybrid control, which is the reason why the hybrid control is less likely to fall when executing fast motions under static stability assumptions.

kinematics for center of body are ill-defined. Three control methods were explored, including 1) a partial IK solution; whole-body solutions using either 2) work-space centering and 3) a hybrid controller which utilizes the partial IK solution for dynamic redundancy resolution. The controllers were tested on several test cases in simulation, designed to force the robot to move dynamically or to the edge of kinematic feasibility. The hybrid controller minimized any violation of static stability assumptions, while also following the commanded trajectories with the least error on all four tests.

Future work will address the implementation issues encountered when trying to run the hybrid control on the real robot, as discussed above. Open loop trajectory generation appears to be a good method to handle this problem. Additionally we will explore using stability metrics as potentials for the COM controller to follow, rather than specifying exact trajectories. For example, one may define a static stability potential which has a flat region over a portion of the support polygon. This is in contrast to the typical notion of the static support margin, the potential function of which would look quadratic (and thus not have a flat region). While the COM is in the flat region, the $[\mathrm{x}, \mathrm{y}]$ coordinates of the COM would essentially not be commanded, and thus would be free to move. This could extend the workspace of the robot, and could alleviate 


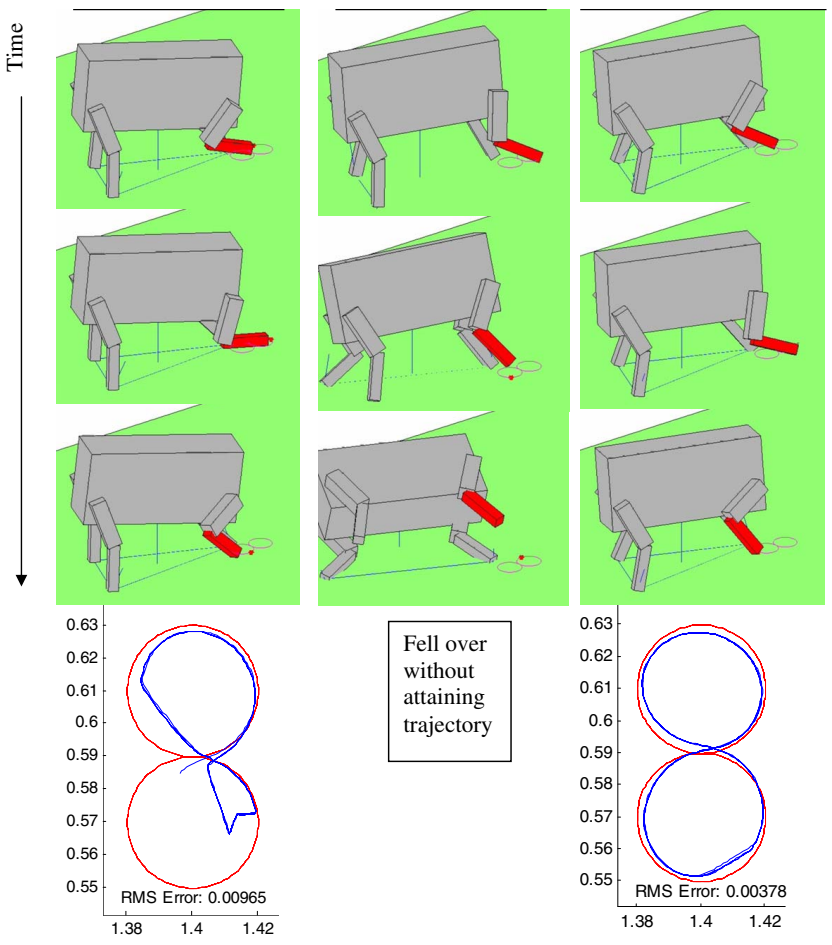

Fig. 4. Simulation Results: Case 3. The red leg designates the swing foot, which is executing a figure- 8 trajectory. Left: Partial IK. Performance is poor particularly because joint limits are reached. Center: Whole-body Jacobian with Work centering. Rapid twisting (jerking) motions cause the robot to loose footing and topple over; the trajectory at this speed can not be achieved with this controller. Right: Hybrid approach. The robot seems to minimize twisting and turning motions, and achieves reasonable trajectory following performance. Bottom: red line is the commanded trajectory (1 second), blue line is the actual trajectory.

higher level controls from having to control the body during a step.

\section{ACKNOWLEDGMENT}

This work was supported by the DARPA Learning Locomotion program (AFRL contract \# FA8650-05-C-7262), and by a Graduate Research Fellowship from the NSF.

\section{REFERENCES}

[1] R. Tedrake, K. Byl, and J. E. Pratt, "Probabilistic stability in legged systems: Metastability and the mean first passage time (FPT) stability margin," In progress, 2006

[2] R. McGhee and A. Frank, "On the stability properties of quadraped creeping gaits," Mathematical Biosciences, vol. 3, pp. 331-351, 1968.

[3] K. Hirai, M. Hirose, Y. Haikawa, and T. Takenaka, "The development of Honda humanoid robot," in Proceedings of the IEEE International Conference on Robotics and Automation (ICRA), 1998, pp. 1321-1326.

[4] Kajita, S. Kanehiro, F. Kaneko, K. Fujiwara, K. Harada, K. Yokoi, K. Hirukawa, and H., "Resolved momentum control: humanoid motion planning based on the linear and angular momentum," Intelligent Robots and Systems (IROS), Proceedings, 2003.

[5] M. B. Popovic, A. Hofmann, and H. Herr, "Zero spin angular momentum control: definition and applicability," Proceedings of the IEEE-RAS/RSJ International Conference on Humanoid Robots, pp. 478-493, 2004.

[6] J. E. Pratt and R. Tedrake, "Velocity based stability margins for fast bipedal walking," in Proceedings of the First Ruperto Carola Symposium on Fast Motions in Biomechanics and Robotics: Optimization and Feedback Control, September 2005.

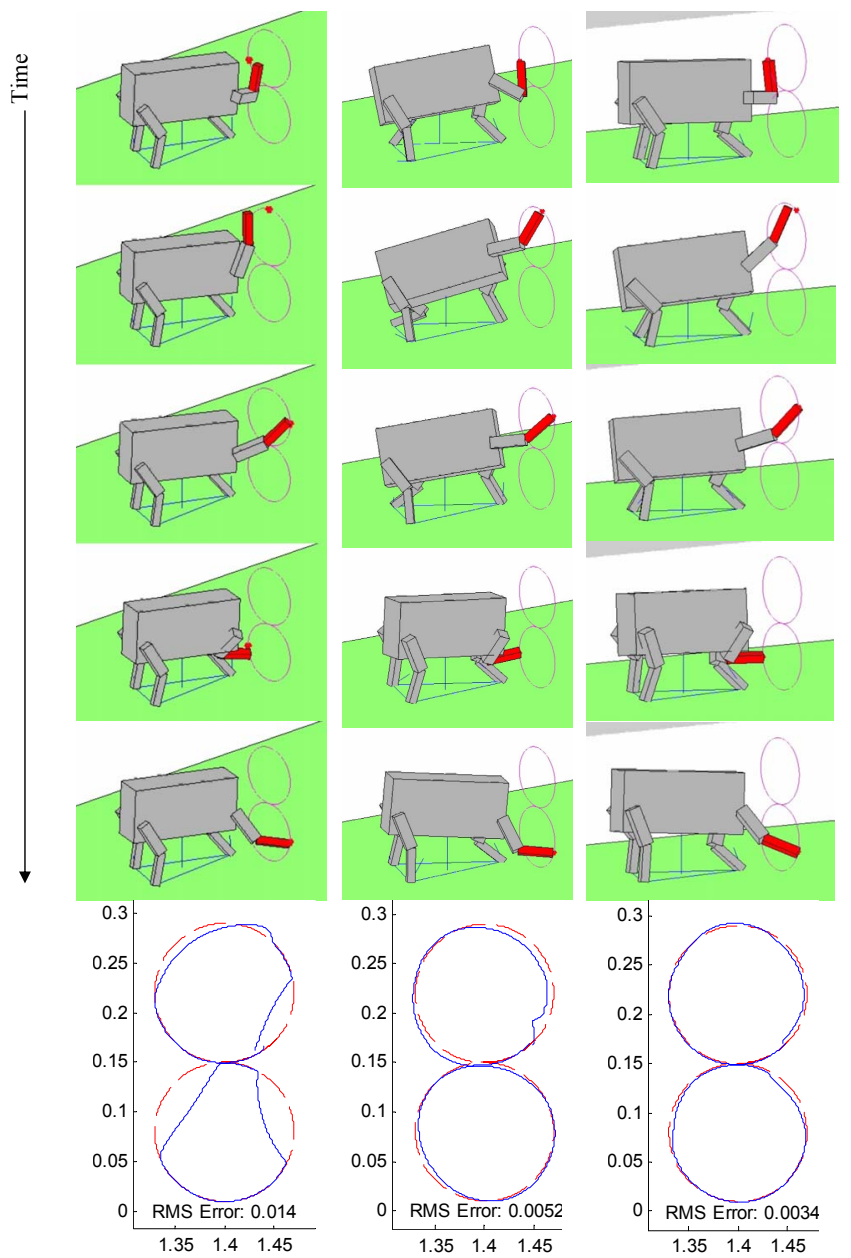

Fig. 5. Simulation Results: Case 4. The red leg designates the swing foot, which is executing a figure-8 trajectory. Left: Partial IK. Performance is poor particularly because joint limits are reached. Center: Whole-body Jacobian with Work centering. Tracking of the figure 8 is good, but COM tracking is affected here, and the robot goes though twisting motions. Right: Hybrid approach. The robot seems to minimize twisting and turning motions, and trajectory following has lowest RMS error. Bottom: red line is the commanded trajectory (15 seconds), blue line is the actual trajectory.

[7] S. Kajita, F. Kanehiro, K. Kaneko, K. Fujiware, K. Harada, K. Yokoi, and $\mathrm{H}$. Hirukawa, "Biped walking pattern generation by using preview control of zero-moment point," in ICRA IEEE International Conference on Robotics and Automation. IEEE, Sep 2003, pp. 1620-1626.

[8] Whitney and D.E., "Resolved motion rate control of manipulators and human prostheses," IEEE Transactions on Man-Machine Systems, vol. 10, no. 2, pp. 47-53, 1969.

[9] Liegeois and A., "Automatic supervisory control of the configuration and behavior of multibody mechanisms," IEEE Trans. Syst. Man Cybern., vol. SMC-7, no. 12, pp. 868 - 871, December 1977, multibody mechanisms;adaptive control;kinematics;robots;manipulators;automatic supervisory control;two level control;

[10] O. Khatib, "A unified approach for motion and force control of robot manipulators: The operational space formulation," IEEE Journal of Robotics and Automation, vol. 3, no. 1, pp. 43-53, February 1987.

[11] O. Khatib, L. Sentis, J. Park, and J. Warren, "Whole-body dynamic behavior and control of human-like robots," International Journal of Humanoid Robotics, vol. 1, no. 1, pp. 29-43, 2004.

[12] J. Nakanishi, R. Cory, M. Mistry, J. Peters, and S. Schaal, "Comparative experimental evaluations of task space control with redundancy resolution," August 2006. 